\title{
Modeling for mung bean variety classification using visible and near-infrared hyperspectral imaging
}

\author{
Chuanqi Xie, Yong He* \\ (College of Biosystems Engineering and Food Science, Zhejiang University, Hangzhou 310058, China)
}

\begin{abstract}
This study was carried out to investigate the feasibility of using visible and near infrared hyperspectral imaging for the variety classification of mung beans. Raw hyperspectral images of mung beans were acquired in the wavelengths of 380-1023 nm, and all images were calibrated by the white and dark reference images. The spectral reflectance values were extracted from the region of interest (ROI) of each calibrated hyperspectral image, and then they were treated as the independent variables. The dependent variables of four varieties of mung beans were set as $1,2,3$ and 4 , respectively. The extreme learning machine (ELM) model was established using full spectral wavelengths for classification. Modified gram-schmidt (MGS) method was used to identify effective wavelengths. Based on the selected wavelengths, the ELM and linear discriminant analysis (LDA) models were built. All models performed excellently with the correct classification rates (CCRs) covering $99.17 \%-99.58 \%$ in the training sets and $99.17 \%-100 \%$ in the testing sets. Fifteen wavelengths (432 nm, $455 \mathrm{~nm}, 468 \mathrm{~nm}, 560 \mathrm{~nm}, 705 \mathrm{~nm}, 736 \mathrm{~nm}, 760 \mathrm{~nm}, 841 \mathrm{~nm}, 861 \mathrm{~nm}, 921 \mathrm{~nm}, 930 \mathrm{~nm}, 937 \mathrm{~nm}, 938 \mathrm{~nm}, 959 \mathrm{~nm}$ and $965 \mathrm{~nm})$ were recommended by MGS. The results demonstrated that hyperspectral imaging could be used as a non-destructive method to classify mung bean varieties, and MGS was an effective wavelength selection method.
\end{abstract}

Keywords: visible and near-infrared hyperspectral imaging, mung bean, classification, modeling, wavelength selection DOI: $10.25165 /$ j.ijabe.20181101.2655

Citation: Xie C Q, He Y. Modeling for mung bean variety classification using visible and near-infrared hyperspectral imaging. Int J Agric \& Biol Eng, 2018; 11(1): 187-191.

\section{Introduction}

Mung bean is welcomed by many people in China, Korea, Burma, India, Japan, Thailand, Pakistan and other Southeast Asian countries due to its high edibleness and medicinal value ${ }^{[1]}$. It contains a lot of nutrients and functional compounds, such as protein, vitamin, lipid, phytochemicals and fiber ${ }^{[2]}$. However, different varieties of mung beans have distinct agronomical, processing and nutritional characteristics ${ }^{[3]}$. Thus, identification of the mung bean variety is of great importance. The current standard methods to differentiate varieties of beans are mainly based on artificial sense and biochemical operation ${ }^{[4,5]}$. However, these methods are inefficient, time-consuming and destructive. Also, professional and qualified technicians are required for such detection methods. Thus, an advanced method (hyperspectral imaging) is needed. This study was carried out to identify the mung bean variety by using the visible and near infrared hyperspectral imaging. Based on the hyperspectral imaging, a multispectral detection system can be designed by the effective wavelengths, which has the potential to be used in industry for online and non-destructive detection.

Hyperspectral imaging combines both spectral and imaging

\section{Received date: 2016-06-08 Accepted date: 2016-09-22}

Biographies: Chuanqi Xie, PhD, Post-doctoral Associate, research interests: crop diseases detection using spectral, hyperspectral imaging and RGB techniques, food engineering, unmanned aerial vehicle, nitrogen nutrition of corn crops. Email: cqxie@zju.edu.cn.

*Corresponding author: Yong $\mathbf{H e}, \mathrm{PhD}$, Professor, research interests: spectral, hyperspectral imaging, unmanned aerial vehicle, internet of things. 866 Yuhangtang Road, Hangzhou 310058, China. Tel/Fax: +86-571-88982143, Email: yhe@zju.edu.cn. techniques together, and has been widely used in previous researches due to its advantages such as being fast, non-destructive, effective and accurate ${ }^{[6-8]}$. Using the hyperspectral imaging system, a spatial map can be created when the sample is scanned by the camera. Each pixel of the hyperspectral image has a spectrum covering the full spectral range. The hyperspectral image (hyperspectral cube) is composed of a series of images at the whole wavelengths, and it contains both spectral and spatial information ${ }^{[9]}$. It can provide a full database with internal and external features of the samples ${ }^{[10]}$, and different varieties of objectives may have various external and internal characteristics, such as color, texture and nutrition content, directly resulting in the spectral signature differently. The spectral signature from a certain pixel of the image is useful for the discrimination and classification of the objectives ${ }^{[11]}$. This technique has been applied in variety classification in many previous studies. The oat and groat kernels were classified using the near-infrared (NIR) hyperspectral imaging system covering the wavelengths of 1006-1650 $\mathrm{nm}^{[12]}$. The partial least squares-discriminant analysis (PLS-DA) model was built for the classification, and three effective wavelengths were identified $(1132 \mathrm{~nm}, 1195 \mathrm{~nm}$ and $1608 \mathrm{~nm})$. Kong et al. ${ }^{[10]}$ identified four cultivars of rice seeds using the NIR hyperspectral imaging in the wavelengths of 1039-1612 nm. PLS-DA, soft independent modeling of class analogy (SIMCA), K-Nearest neighbor $(\mathrm{KNN})$, support vector machine (SVM) and random forest (RF) were established to identify the cultivars. Twelve useful wavelengths (1069 nm, $1079 \mathrm{~nm}, 1139 \mathrm{~nm}, 1167 \mathrm{~nm}, 1183 \mathrm{~nm}, 1227 \mathrm{~nm}, 1281 \mathrm{~nm}$, $1304 \mathrm{~nm}, 1328 \mathrm{~nm}, 1389 \mathrm{~nm}, 1467 \mathrm{~nm}$ and $1558 \mathrm{~nm})$ were selected by weighted regression coefficients method. Kamruzzaman et al. ${ }^{[13]}$ used the NIR hyperspectral imaging to 
discriminate lamb muscles. Principal component analysis (PCA) was used to compress the dimensionality, and six wavelengths (934 nm, $974 \mathrm{~nm}, 1074 \mathrm{~nm}, 1141 \mathrm{~nm}, 1211 \mathrm{~nm}$ and $1308 \mathrm{~nm}$ ) were determined by PCA loadings. Quality classification of cooked and sliced hams was also studied using NIR hyperspectral imaging ${ }^{[14]}$. PCA was used for selecting effective wavelengths, and eight wavelengths $(980 \mathrm{~nm}, 1061 \mathrm{~nm}, 1141 \mathrm{~nm}, 1174 \mathrm{~nm}$, $1215 \mathrm{~nm}, 1325 \mathrm{~nm}, 1436 \mathrm{~nm}$ and $1641 \mathrm{~nm}$ ) were identified to discriminate different turkey ham qualities. Barbin et al. ${ }^{[15]}$ investigated the identification of fresh and frozen-thawed meat by using the NIR hyperspectral imaging. The PLS-DA model achieved the correct classification of $100 \%$ based on the effective wavelengths recommended by weighted regression coefficients method. Wei et al. ${ }^{[16]}$ discriminated the ripeness (unripe, mid-ripe, ripe and over-ripe) of persimmon fruit using the visible and near-infrared hyperspectral imaging (400-1000 nm). Three classification models (linear discriminant analysis (LDA), SIMCA and least squares support vector machines (LS-SVM)) were built to classify the different types of persimmons. Three wavelengths $(518 \mathrm{~nm}, 711 \mathrm{~nm}$ and $980 \mathrm{~nm})$ were selected by successive projection algorithm (SPA). Based on the spectral and texture feature at the three wavelengths, the LDA model obtained the best correct classification rate of $95.30 \%$. Deng et al. ${ }^{[17]}$ used spectral angle mapping (SAM) model combined with hyperspectral images to distinguish weeds from background and cabbages. All of these studies demonstrated that the hyperspectral imaging has the potential to be used for variety classification.

The aim of this study was to develop an effective method to classify mung bean varieties using the hyperspectral imaging. The specific objectives were to: (1) classify different varieties of mung beans based on spectral reflectance information; (2) identify effective wavelengths that play the most significant roles for the variety classification using modified gram-schmidt (MGS); and (3) compare the performance of different classification models.

\section{Materials and methods}

\subsection{Work flow}

The process of this study can be seen in Figure 1. In the first step, raw hyperspectral images of four varieties of mung beans were acquired by the visible and near infrared hyperspectral imaging camera. All raw hyperspectral images were then corrected by the white and dark reference images. Spectral reflectance values were extracted from the corrected hyperspectral images and treated as the dependent variables. All samples were divided into two sets (training and testing) at a ratio of 2:1. One classification model (extreme learning machine, ELM) was established based on the full spectral wavelengths. Then, MGS method was used to select effective wavelengths. Based on these selected wavelengths, the ELM and LDA models were built, respectively. The optimal model was finally determined by the values of correct classification rates (CCRs).

\subsection{Samples}

Four different brands of mung beans (Jinlongyu, Ouyi, Saiwengfu and Xiangmanyuan) were used in this study. A volume of $60 \mathrm{~cm}^{3}$ of mung beans were evenly distributed in a glass dish $(d=90 \mathrm{~mm})$. For each brand, there were 90 dishes. Each dish represented one sample, and was then scanned individually by the hyperspectral imaging system. Finally, a total of 360 samples for the four varieties were used in this study.

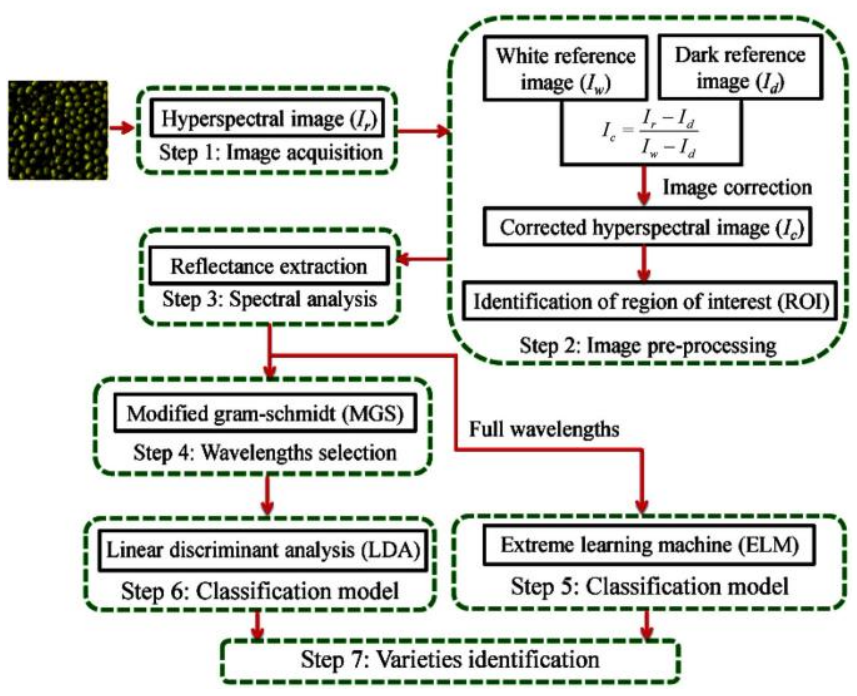

Figure 1 Flow chart of this study

\subsection{Experiment device}

A hyperspectral imaging system which covers the spectral wavelengths of 380-1023 nm was used in this study (Figure 2). This system consisted of a CCD camera (C8484-05, Hamamatsu City, Japan), a lens (OLE-23, USA), an imaging spectrograph (V10E, Specim, Oulu, Finland), two light sources (Oriel Instruments, USA) provided by two $150 \mathrm{~W}$ quartz tungsten halogen lamps, a computer, and a conveyer belt operated by a stepper motor (IRCP0076, Isuzu Optics Corp., Taiwan, China). The area CCD array detector has $672 \times 512$ (spatial $\times$ spectral) pixels, and the spectral resolution is $2.8 \mathrm{~nm}$. Samples were scanned by the hyperspectral imaging system line by line, and the reflected light was dispersed by the spectrograph and captured by the area CCD array detector in spatial-spectral axes. A hyperspectral image can be generated when the sample is scanned by the camera. Each hyperspectral image contains hundreds of gray images, and each pixel contains both spectral and spatial features (Figure 3). The ENVI 4.7 software (Research System Inc., Boulder, Co., USA) was used for obtaining spectral reflectance information from hyperspectral images. MATLAB R2009a (The Math Works Inc., Natick, MA, USA) was used to identify effective wavelengths and establish classification models.

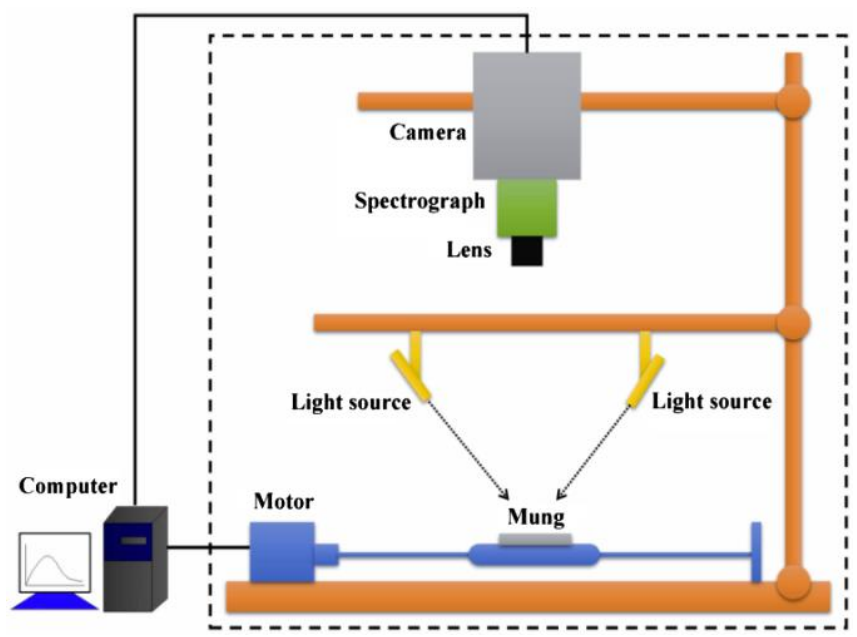

Figure 2 Visible and near-infrared hyperspectral imaging system 

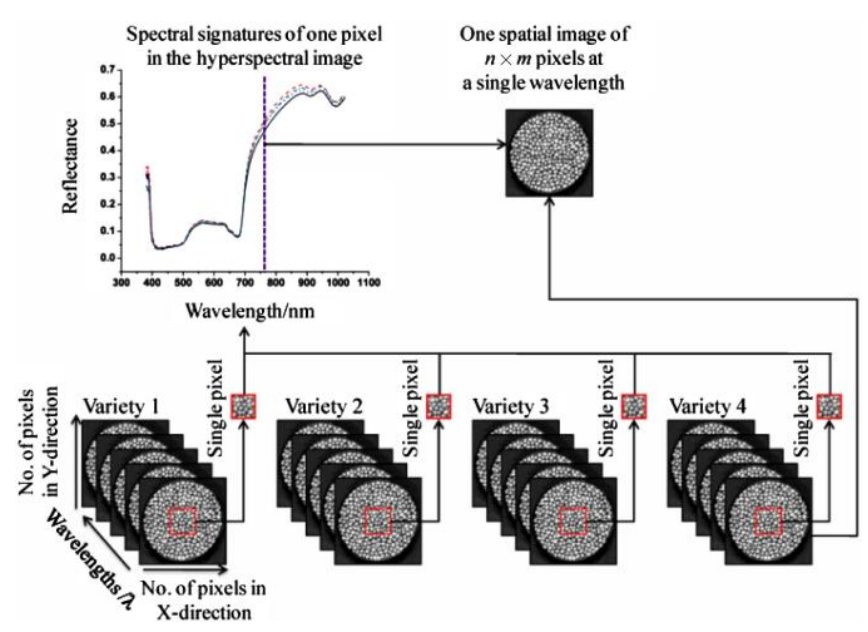

Figure 3 Hyperspectral imaging of mung beans

\subsection{Images acquisition and correction}

In order to get an image without distortion and overexposure, the hyperspectral imaging camera should be tested several times for obtaining the best suitable exposure time and moving speed. Finally, the two parameters were $0.09 \mathrm{~s}$ and $3.0 \mathrm{~mm} / \mathrm{s}$, respectively. A dark reference image with the reflectance of $0 \%$ was acquired by turning off the light and covering the lens with its cap. The white reference image with the reflectance of about $99 \%$ was obtained by scanning a white Teflon board (CAL-tile200, $200 \mathrm{~mm} \times 25 \mathrm{~mm} \times$ $10 \mathrm{~mm}$, Isuzu Optics Corp., Taiwan, China). Then, each glass dish with mung beans was put on the conveyer belt to be scanned by the hyperspectral imaging system in sequence. A raw hyperspectral image (hyperspectral cube) with a dimension of $(x, y, \lambda)$ was created as the sample was scanned along the direction of the $y$ dimension. The hyperspectral image had 672 pixels in the $y$ dimension and 512 bands in the $\lambda$ dimension. When the raw hyperspectral image was generated, it should be corrected with the dark and white reference images based on the Equation (1):

$$
I_{c}=\frac{I_{r}-I_{d}}{I_{w}-I_{d}}
$$

where, $I_{r}$ is the raw hyperspectral image; $I_{d}$ is the dark reference image; $I_{w}$ is the white reference image, and $I_{c}$ is the corrected hyperspectral image.

\subsection{Spectral reflectance extraction}

After images acquisition and correction, an area (region of interest, ROI) with $50 \times 50$ pixels was cropped from each corrected hyperspectral image, and spectral reflectance values of all pixels extracted from the ROI were averaged and treated as the independent variables. The dependent variables for the four varieties were set as 1 (Jinlongyu), 2 (Ouyi), 3 (Saiwengfu) and 4 (Xiangmanyuan), respectively. After all samples were sorted according to the variety value, one sample was then randomly picked out from every three ones ${ }^{[18]}$, resulting in 240 samples for the training set and 120 ones for the testing set.

\subsection{Classification models}

In order to compare the performance of different models and simultaneously identify the optimal one, two classification models were used in this study. ELM, which has a good generalization performance for feed forward neural networks, was applied in building classification and regression models in previous studies $^{[19,20]}$. It can effectively solve problems such as local minima and over-fitting ${ }^{[21]}$. The learning speed of ELM is faster than that of traditional feed forward network such as the back-propagation (BP) algorithm ${ }^{[22]}$. LDA is a supervised recognition algorithm, and can be used to find a linear combination of features that can classify different varieties of objectives $^{[16]}$. The LDA algorithm produces a number of orthogonal linear discriminant functions which makes the samples to be classified in different categories ${ }^{[23]}$. Both classification models were built using MATLAB R2009a software.

\subsection{Wavelength selection}

The spectral feature, which covers the wavelengths from $380 \mathrm{~nm}$ to $1023 \mathrm{~nm}$, usually contains highly redundant information among different wavelengths ${ }^{[24]}$. Thus, the selection of effective wavelengths is a crucial step in spectral analysis ${ }^{[25]}$. The final aim of selecting effective wavelengths is to establish a subset of spectral wavelengths to replace the whole ones. The selected wavelengths can be equal to or even more effective than the full wavelengths ${ }^{[26]}$. Also, these wavelengths can not only simplify the model but also be used to develop a multispectral imaging classification system. MGS was used to select useful wavelengths in this study.

The principle of MGS is to build a new orthogonal basis based on the original one by projection. An eigenvector, which is selected from the feature matrix $X=\left[x_{1}, x_{2}, \ldots, x_{n}\right]$, can be treated as the first orthogonal basis $x_{z 1}$. Then the projection of other vector quantities onto this orthogonal basis can be calculated $\left(x_{\text {proji }}=\frac{\left(x_{i}, x_{z 1}\right)}{\left\|x_{z 1}\right\|^{2}} x_{z 1}\right)$. Making the difference value $\left(x_{i}{ }^{\prime}=x_{i}-x_{\text {proji }}\right)$ obtained from other vector quantities and this projection and $x_{z 1}$ orthogonal, resulting in $n-1$ eigenvectors of the first orthogonal basis. Similarly, the projection of the class label vector $Y$ onto the first orthogonal basis $\left(Y_{p r o j i}=\frac{\left(Y, x_{z 1}\right)}{\left\|x_{z 1}\right\|^{2}} x_{z 1}\right)$ can be calculated. Make the difference value obtained from $Y$ and projection and $x_{z 1}$ orthogonal. Finally, the eigenvector can be identified. The main procedures can be seen in Table $1^{[27]}$.

Step 1: Each eigenvector of characteristic matrix $X=\left[x_{1}, x_{2}, \ldots, x_{n}\right]$ is normalized, $x_{n i}=\frac{x_{i}}{\left\|x_{i}\right\|}(i=1,2, \ldots n)$;

Step 2: The inner product of class label vector $Y$ and each normalized eigenvector is calculated $y_{\text {proj } i}=\left(Y \cdot x_{n i}\right)(i=1,2, \ldots n)$, the eigenvector $x_{n s}(s=1,2, \ldots n)$ that maximizes $y_{\text {proj_i }}$ is selected as normalized orthogonal basis;

Step 3: The eigenvector of feature matrix $X=\left[x_{1}, x_{2}, \ldots, x_{n}\right]$ is projected onto normalized orthogonal basis $x_{n s}, x_{p r o j i}=\frac{\left(x_{i}, x_{n s}\right)}{\left\|x_{n s}\right\|^{2}} x_{n s}$, the difference value $\left(x_{i}{ }^{\prime}=x_{i}-x_{\text {proji }}\right)$ of eigenvector and projection is calculated;

Step 4: The class label vector $Y$ is projected onto normalized orthogonal basis $x_{n s}, Y_{m p r i}=\frac{\left(Y, x_{n s}\right)}{\left\|x_{n s}\right\|^{2}} x_{n s}$, the difference value $\left(Y^{\prime}=\right.$ $\left.Y-Y_{\text {proji }}\right)$ of $Y$ and projection is calculated;

Step 5: $X=\left[x_{1}^{\prime}, \ldots x_{s-1}^{\prime}, x_{s+1}^{\prime}, \ldots, x_{n}^{\prime}\right], Y=Y, n=n-1$;

Step 6: Calculation will stop when the required eigenvectors are obtained, or it will return to step 2 .

This method belongs to the backward feature selection, which executes the selection by removing one or more feature from the whole features. The backward feature selection method can keep 
the informative features as many as possible. On the other hand, MGS considers the relationship between the feature structures and label information, which can improve the performance of the subsequent classification or prediction model. As a whole, the merits of MGS can be summarized as follows: (1) backward feature selection, and (2) the feature selection combines the feature structure and label information.

\section{Table 1 Main procedures of modified gram-schmidt (MGS)} algorithm

\begin{tabular}{|c|c|}
\hline Input & Feature matrix $X=\left[x_{1}, x_{2}, \ldots, x_{n}\right]$, class label vector $Y$ \\
\hline Output & feature matrix $X s$ selected by MGS \\
\hline 1 & for $k=1$ :featnum \%Main loop \\
\hline 2 & $x_{n i}=\frac{x_{i}}{\left\|x_{i}\right\|} \%$ Normalized feature matrix \\
\hline 3 & $\begin{array}{l}x_{n s}=\operatorname{argmax}\left(Y, x_{n i}\right), X_{s}(k)=x_{n s} \quad \% \text { Finding the direction of } \\
\text { maximum inner product }\end{array}$ \\
\hline 4 & $x_{i}^{\prime}=x_{i}-\frac{\left(x_{i}, x_{n s}\right)}{\left\|x_{n s}\right\|^{2}} x_{n s} \quad \%$ Projecting $X$ onto $x_{n s}$, computing the residual $X$ \\
\hline 5 & $Y^{\prime}=Y-\frac{\left(Y, x_{w s}\right)}{\left\|x_{n u}\right\|^{2}} x_{w} \quad \%$ Projecting $Y$ onto $x_{n s}$, computing the residual $Y$ \\
\hline 6 & $\begin{array}{l}X=\left[x_{1}^{\prime}, \ldots x_{s-1}^{\prime}, x_{s+1}^{\prime}, \ldots, x_{n}^{\prime}\right], Y=Y^{\prime}, n=n-1 \% \text { Changing the variables to } \\
\text { iterate }\end{array}$ \\
\hline 7 & end \\
\hline 8 & return $x_{s}$ \\
\hline
\end{tabular}

\section{Results and discussion}

\subsection{Spectral feature analysis}

The mean spectral reflectance curves of the four varieties of mung beans were shown in Figure 4 . It can be seen that the general trends of the spectral reflectance curves of different varieties were very similar with some noise at the beginning of the wavelength, and some peaks as well as valleys covering the whole wavelengths. Different peaks and valleys are corresponding to various spectral bands, for example, the wavelength at $970 \mathrm{~nm}$ indicates there is a water absorption band here. Also, obvious difference can be seen in the spectral region of 700-1023 nm, which means most of the effective wavelengths for the classification should be located in this region. In order to minimize the influence of the noise, the wavelengths at the beginning were rejected, resulting in spectral wavelengths of 395-1023 nm (band 14-band 512) being used in this study.

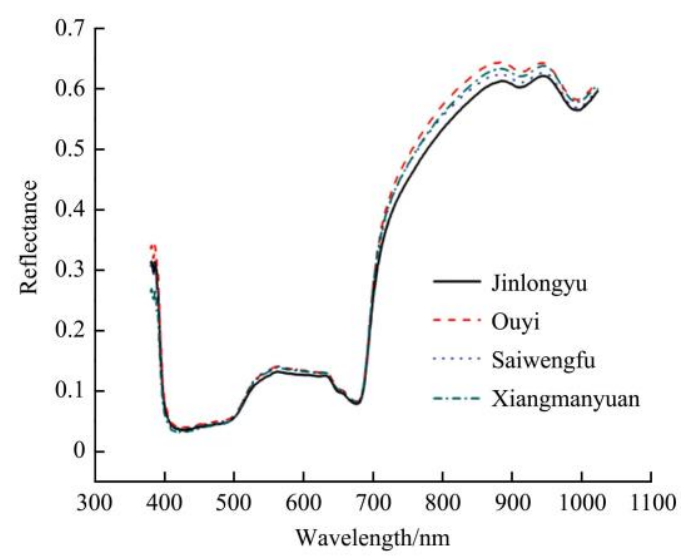

Figure 4 Average spectral reflectance curves of the four varieties of mung beans

\subsection{Classification based on whole wavelengths}

In this study, the ELM classification model was firstly established based on full spectral wavelengths. The reflectance values were treated as $X$ variables, and the varieties were treated as $Y$ variables (Jinlongyu-1, Ouyi-2, Saiwengfu-3, Xiangmanyuan-4). The ELM model obtained a satisfying result with CCRs of $99.58 \%$ in the training set and $100 \%$ in the testing set. No samples were incorrectly identified in the testing set. However, the large number of input variables will increase the calculation time and cannot be used for designing a multispectral classification system. Therefore, effective wavelengths should be identified for establishing simplified classification models.

\subsection{Effective wavelengths}

In order to improve the performance of classification models and simplify the calculation, MGS was carried out to identify the key wavelengths. Based on the selected wavelengths, the spectral data set was then reduced into a matrix with a dimension of $m \times x$ ( $m$ was the number of samples, and $x$ was the number of selected wavelengths). As a result, a total of fifteen wavelengths (432 nm, $455 \mathrm{~nm}, 468 \mathrm{~nm}, 560 \mathrm{~nm}, 705 \mathrm{~nm}, 736 \mathrm{~nm}, 760 \mathrm{~nm}, 841 \mathrm{~nm}, 861$ $\mathrm{nm}, 921 \mathrm{~nm}, 930 \mathrm{~nm}, 937 \mathrm{~nm}, 938 \mathrm{~nm}, 959 \mathrm{~nm}$ and $965 \mathrm{~nm}$ ) were obtained. Compared with full spectral wavelengths, the number of selected wavelengths was greatly reduced. It only accounted for $3.01 \%$ of the number of whole wavebands (band 14-band 512). These selected wavelengths were then used as the new input variables for establishing classification models.

\subsection{Classification based on selected wavelengths}

The ELM and LDA models were then built using the selected wavelengths suggested by MGS (Table 2). MGS-ELM model performed excellently with the CCRs of $99.17 \%$ in both training and testing sets. MGS-LDA model acquired the CCRs of $99.17 \%$ in the training set and $98.33 \%$ in the testing set. Compared with the full wavelengths-based ELM model, there was a little decrease of CCR in the testing set of MGS-ELM model. However, the result was still excellent (99.17\%). Also, the number of input variables were largely reduced, which made the classification model simple. It indicated that these wavelengths selected by MGS were very useful for classifying different varieties of mung beans. As mentioned above, the spectral reflectance curves were obviously different in the wavelengths of 700-1023 nm. Most of the selected wavelengths were located in this region. This might be why the selected wavelengths-based models could also obtain good classification results.

Table 2 Classification results by using different models

\begin{tabular}{|c|c|c|c|c|c|c|c|}
\hline \multirow{2}{*}{$\begin{array}{l}\text { Classification } \\
\text { model }\end{array}$} & \multirow{2}{*}{$\begin{array}{c}\text { Number of } \\
\text { wavelengths }\end{array}$} & \multicolumn{3}{|c|}{ Training set } & \multicolumn{3}{|c|}{ Testing set } \\
\hline & & No. & Missed & $\begin{array}{c}\mathrm{CCR}^{\mathrm{a}} \\
1 \%\end{array}$ & No. & Missed & $\begin{array}{c}\mathrm{CCR}^{\mathrm{a}} \\
1 \%\end{array}$ \\
\hline MGS-ELM & 15 & 240 & 2 & 99.17 & 120 & 1 & 99.17 \\
\hline MGS-LDA & 15 & 240 & 2 & 99.17 & 120 & 2 & 98.33 \\
\hline
\end{tabular}

\section{Conclusions}

This study demonstrated that the visible and NIR hyperspectral imaging could be used to classify the four different varieties of mung beans. MGS was efficient for selecting useful wavelengths. The numbers of selected wavelengths suggested by MGS only accounted for $3.01 \%$ of that of the full wavelengths. The full wavelengths-based ELM model obtained an excellent result with a very high CCR value (100\%) in the testing set. Those models established based on selected wavelengths obtained prominent results with CCRs ranging from $98.33 \%$ to $99.17 \%$ in the testing sets. Although the results acquired by selected wavelengths were a little lower than that obtained by full wavelengths, they are still excellent. Moreover, a large amount of the full wavelengths were 
reduced into a few ones, which have the potential to be used for designing an online detection system. However, in order to get more robust results, more samples and varieties should be considered in further studies.

\section{Acknowledgements}

This work was supported by the National Key Scientific Instrument and Equipment Development Projects (2014YQ470377), the Scientific Research Foundation for Returned Overseas Students and the Fundamental Research Funds for the Central Universities of China (2012FZA6005, 2013QNA6011).

\section{[References]}

[1] Zhang X, Shang P, Qin F, Zhou Q, Gao B, Huang H, et al. Chemical composition and antioxidative and anti-inflammatory properties of ten commercial mung bean samples. LWT-Food Science and Technology, 2013; 54(1): 171-178.

[2] Huang X Y, Cai J R, Xu B J. Kinetic changes of nutrients and antioxidant capacities of germinated soybean (Glycine max L.) and mung bean (Vigna radiata L.) with germination time. Food Chemistry, 2014; 143: 268-276.

[3] Dahiya P K, Linnemann A R, Nout M J R, van Boekel M A J S, Grewal R B. Nutrient composition of selected newly bred and established mung bean varieties. LWT-Food Science and Technology, 2013; 54(1): 249-256.

[4] Tantasawat P, Trongchuen J, Prajongjai T, Seehalak W, Jittayasothorn Y. Variety identification and comparative analysis of genetic diversity in yardlong bean (Vigna unguiculata spp. sesquipedalis) using morphological characters, SSR and ISSR analysis. Scientia Horticulturae, 2010; 124(2): 204-216.

[5] Sun J, Jiang S Y, Mao H P, Wu X H, Li Q L. Classification of black beans using visible and near infrared hyperspectral imaging. International Journal of Food Properties, 2016; 19(8): 1687-1695.

[6] Tao F F, Peng Y K, Li Y Y. Feature extraction method of hyperspectral scattering images for prediction of total viable count in pork meat. Int $\mathrm{J}$ Agric \& Biol Eng, 2015; 8(4): 95-105.

[7] Liu Y L, Lyu Q, He S L, Yi S L, Liu X F, Xie R J, et al. Prediction of nitrogen and phosphorous contents in citrus leaves based on hyperspectral imaging. Int J Agric \& Biol Eng, 2015; 8(2): 80-88.

[8] Xie C Q, He Y, Li X L, Liu F, Du P P, Feng L. Study of detection of SPAD value in tomato leaves stressed by grey mold based on hyperspectral technique. Spectroscopy and Spectral Analysis, 2012; 32(12): 3324-3328.

[9] Xie C Q, Li X L, Nie P C, He Y. Application of time series hyperspectral imaging (TS-HSI) for determining water content within tea leaves during drying. Transactions of the ASABE, 2013; 56(6): 1431-1440.

[10] Kong W W, Zhang C, Liu F, Nie P C, He Y. Rice seed cultivar identification using near-infrared hyperspectral imaging and multivariate data analysis. Sensors, 2013; 13(7): 8916-8927.

[11] Kim I, Xu C Z, Kim M S. Poultry skin tumor detection in hyperspectral images using radial basis probabilistic neural network. Advances in Neural Networks-ISNN, 2006; 3973: 770-776.

[12] Serranti S, Cesare D, Marini F, Bonifazi G. Classification of oat and groat kernels using NIR hyperspectral imaging. Talanta, 2013; 103: 276-284.

[13] Kamruzzaman M, Elmasry G, Sun D W, Allen P. Application of NIR hyperspectral imaging for discrimination of lamb muscles. Journal of Food Engineering, 2011; 104(3): 332-340.

[14] ElMasry G, Iqbal A, Sun D W, Allen P, Ward P. Quality classification of cooked, sliced turkey hams using NIR hyperspectral imaging system. Journal of Food Engineering, 2011; 103(3): 333-344.

[15] Barbin D F, ElMasry G, Sun D W. Non-destructive assessment of microbial contamination in porcine meat using NIR hyperspectral imaging. Food Science \& Emerging Technologies, 2013; 18: 226-236.

[16] Wei X, Liu F, Qiu Z J, Shao Y N, He Y. Ripeness classification of astringent persimmon using hyperspectral imaging technique. Food and Bioprocess Technology, 2014; 7(5): 1371-1380.

[17] Deng W, Huang Y B, Zhao C J, Wang X. Identification of seedling cabbages and weeds using hyperspectral imaging. Int J Agric \& Biol Eng, 2015; 8(5): 65-72.

[18] Li X L, Xie C Q, He Y, Qiu Z J, Zhang Y C. Characterizing the moisture content of tea with diffuse reflectance spectroscopy using wavelet transform and multivariate analysis. Sensors, 2012; 12(7): 9847-9861.

[19] Pal M. Extreme-learning-machine-based land cover classification International Journal of Remote Sensing, 2009; 30(14): 3835-3841.

[20] Ouyang Q, Chen Q S, Zhao J W, Lin T. Determination of amino acid nitrogen in soy sauce using near infrared spectroscopy combined with characteristic variables selection and extreme learning machine. Food and Bioprocess Technology, 2013; 6(9): 2486-2493.

[21] Zhu Q Y, Qin A K, Suganthan P N, Huang G B. Evolutionary extreme learning machine. Pattern Recognition, 2005; 38(10): 1759-1763.

[22] Huang G B, Zhu Q Y, Siew C K. Extreme learning machine: Theory and applications. Neurocomputing, 2006; 70(1): 489-501.

[23] Riovanto R, Cynkar W U, Berzaghi P, Cozzolino D. Discrimination between Shiraz wines from different Australian regions: The role of spectroscopy and chemometrics. Journal of Agricultural and Food Chemistry, 2011; 59(18): 10356-10360.

[24] ElMasry G, Sun D W, Allen P. Near-infrared hyperspectral imaging for predicting colour, $\mathrm{pH}$ and tenderness of fresh beef. Journal of Food Engineering, 2012; 110(1): 127-140.

[25] Barbin D F, ElMasry G, Sun D W, Allen P. Predicting quality and sensory attributes of pork using near-infrared hyperspectral imaging. Analytica Chimica Acta, 2012; 719: 30-42.

[26] Xie C Q, Li X L, Shao Y N, He Y. Color measurement of tea leaves at different drying periods using hyperspectral imaging technique. Plos One, 2014; 9(12): e113422.

[27] Amsterdam W $\mathrm{H}$. Iterative algorithms for gram-schmidt orthogonalization. Computing, 1989; 41(4): 335-348. 